DOE $|E R| 25006-6$

\title{
Computing Environments,
} Interactive Graphics and Nonparametric Methods for Data Analysis (Grant DE-FG06-75-ER25006 A003) Progress Report 1990-91 Research Plan 1991-92

\author{
John Alan McDonald \\ Finbarr O'Sullivan \\ Werner Stuetzle \\ Department of Statistics \\ University of Washington \\ Seattle, WA 98195
}

March 11, 1992

\section{DISCLAIMER}

\begin{abstract}
This report was prepared as an account of work sponsored
Government. Neither the United States Gork sponsored by an agency of the United States employees, makes any warranty, express or implied, nor any agency thereof, nor any of their process disc accuracy, completeness, or usefulness of assumes any legal liability or responsience herein to any represents that its use would not infringrmation, apparatus, product, or manufacturer, or otherwic commercial product, process, or service by owned rights. Refermendation, or favoring by does not necessarily constitute or imply trade name, trademark, and opinions of authors the United States Government or anyly its endorsement, recomUnited States Government or ansed herein do not necessarily state or thereof. The views United States Government or any agency thereof.
\end{abstract}




\section{Introduction}

This report reflects progress of work under the grant up the end of 1991 . It is structured as follows. Sections $2-6$ describe work by J.A. McDonald and W. Stuetzle. Sections 7 and 8 list lectures at conferences given by J.A. McDonald and W.Stuetzle, and their publications. Section 9 summarizes the research performed by Finbarr D'Sullivan, and lists his publications and lectures. Sections 10-12 summarize the research by graduate students Kevin Anderson, Michael Sannella, and Steve McKinney. Finally, section 13 contains the bibliography.

As $1990 / 91$ has been the last year of the grant, we do not submit an extensive research plan. We will use the year 1991/92, for which we have been granted a no cost extension, to continue working on research in the general area supported by the grant, to wrap up whatever loose ends remain, to bring the Ph.D. theses of Sannella and McKinney to conclusion, and to prepare a proposal for future work.

\section{The Arizona data visualization system}

Arizona is intended to be a portable, public-domain collection of tools supporting scientific computing, quantitative graphics, and data analysis, implemented in Common Lisp and CLOS (the Common Lisp Object System) [89].

Discussion of the philosophy underlying Arizona can be found in $[69,70,57$, $71,59,91,901$. Briefly, the design is motivated by our belief that an ideal system for scientific computing and data analysis should have:

- One language that can be used for both for line-by-line interaction or defining compiled procedures.

- Minimal overhead in adding new compiled procedures (or other definitions).

- A language thit supports a wide variety of abstractions and the definition of new kinds of abstractions.

- Programming tools (editor, debugger, browsers, metering and monitoring tools). 
- Automatic memory management (dynamic space allocation and garbage collection).

- Portability over many types of workstations and operating systems.

- A community of users and developers.

- Access to traditional Fortran scientific subroutine libraries or equivalents.

- A representation of scientific data directly in the data structures of the language.

- Comprehensive numerical, graphical, and statistical functionality.

- Device independent static output graphics.

- Window based interactive graphics.

- Support for efficient and concurrent access to large databases.

- Documentation and tutorials, both paper and on-line.

One reason Arizona is based on Common Lisp is that the first nine points (through "access to Fortran") come for free with standard Common Lisp environments. The remaining six are the research aspects of Arizona.

Arizona is divided into a number of modules with limited interdependencies, to permit individual modules to stabilize and be "released" and used independently. The word "module" is used here informally. The definitions in a module will usually be in a package defined for that module and the source code for those definitions will usually be found in files in a single directory. A number of the modules in Arizona are described below. Current modules in alpha release are Arizona-Tools, Definitons, Announcements, Actors, Geometry, Slate, Chart, Graph, Browser, and an interface between lisp and the Fortran optimization package NPSOL. Four other modules have seen some use but are likely to have more significant changes before any sort of release: Clay, Basic-Math, Probability, and Cactus.

\subsubsection{Arizona-Tools}

The Arizona-Tools module [74] contains assorted general purpose utilities for Common Lisp programming, which don't have an obvious home in any of 
the other modules. Collecting them in one place saves users from reinventing many minor variations of the same wheel and encourages programming style consistent with the conventions suggested in [74]. For example, ArizonaTools defines protocols for copying, saving to file, and destroying CLOS objects, and default implementations of those protocols, which are some essential facilities for a primitive database using persistent CLOS objects.

\subsubsection{Definitions}

The Definitions module [61] provides the beginnings of a database for Common Lisp source code objects. The major use of this database at present is in automatically typesetting reference manuals (using Latex [48]), examples of which are the reference manual sections of $[74,61,60,75,77,76,68,62$, $66,63,73]$. It is inspired in part by the Definition Groups of Bobrow et. al [8] and the USER-MANUAL of Kantrowitz [47].

The primary purpose of Definitions is to make possible convenient runtime access to information available in Common Lisp source code, that is lost in the normal process of reading, evaluating, and/or compiling. Evaluating some Lisp definitions, such as defclass, results in a first class Lisp object with a reasonable and reasonably portable protocol for extracting useful information, such as the direct sub- and super-classes. However, most Lisp definitions, such as defun, while producing identifiable objects, have limited facilities for extracting useful information; usually the documentation string is all that is available. And other definitions, such as defstruct, do not even produce an identifiable object.

The Definitions module provides functions to read source files and create a Definition object for each lisp definition in those files, retaining the complete original defining lisp form. The protocol for instantiated Definition objects consists a large number of functions for parsing and extracting useful information from the defining forms, a few functions for filtering and sorting sequences of Definitions, and a few functions implementing a standard format for printing Definitions to a Latex file.

\subsubsection{Announcements}

The Announcements module [68] provides a simple mechanism for maintaining dependencies between objects through message broadcasting, similar to the Field [85] and Forest [33] environments. It is derived from a Com- 
mon Lisp implementation of Events/Mediators [93] by Mark Niehaus for the Prism system [45]. Like Field and Forest, it can be thought of as a mechanism for implicit invocation as discussed in [34, 94].

The basic idea is to allow an object (the announcer to notify other objects (the audience) of a change of state (or some other event) without the announcer having to know of which objects need to be notified of which events.

An announcement is defined with a call to defannouncement. Any announcer object can announce any defined announcement, which results in every member of the audience for that announcer-announcement pair being notified. Any object can join the audience for a particular announcer-announcement pair with a call to register.

\subsubsection{Actors}

The Actors module [63] is primarily intended as a mechanism for interpreting and distributing asynchronous (input) events in a multiprocessing Lisp environment. It is inspired in part by the actor message passing paradigm of Carl Hewitt (as described in [1]).

An Actor is an object with a message queue; each actor handles its messages in its own, separate, thread of computation, at least conceptually in parallel with other actors and in parallel with any read-eval-print loops or other $\mathrm{I} / \mathrm{O}$ processes that may exist.

Actors contain multiple Actor-Role objects; the current role determines how an actor handles a message at any given time. A common response to a. message is to simply change the current role. An actor can be thought of as an implementation of a finite state machine for handling (input) events; each role can be thought of as a state in a state-transition diagram and the events that cause changes of role are the transitions.

Actually, a role is more accurately thought of as a fairly independent subgraph of a state-transition diagram. The advantage of encapsulating moreor-less independent chunks of behavior in role objects is that they can then be composed in different ways in various subclasses of Actor, making it easior to implement new graphical user interfaces.

One important subclass of Actor is Interactor, in the Slate package. Interactors are actors whose queves receive messages corresponding to the low level mouse and keyboard events received by windows.

Another important subclass is Coordinator, in the Clay package. Coordinators are objects that represent and maintain relationships between interac- 
tive diagrams and the diagrams' subject - the underlying data that the diagram represents. "Coordinator" is really just another word for "Mediator" as used in [93]. Coordinators receive messages from diagrams requesting operations to be performed on their subjects (which originate from user input) and they also receive messages (via Announcements [68]) from subjects that indicate that diagrams need to be updated to reflect changes in the state of a subject.

\subsubsection{Geometry}

The Geometry module [75] is intended to support common geometric calculations arising in graphics, numerical linear algebra, optimization, and scientific computing in general. The goal is to allow geometric computation using abstractions that directly represent high level mathematical concepts like affine spaces, vectors, and linear transformations, while retaining the level of performance provided by traditional scientific subroutine packages like Linpack[24]. It will be derived from Cactus $[72,59]$ and improved with ideas from work by DeRose [20, 21, 22] and Segal [88].

However, the current release of the Geometry module does not support general geometric calculations. It consists of two submodules specialized for high performance in simple graphics calculations, needed by the Slate and Chart modules discussed below. The Screen Geometry submodule supports calculations in a discrete two-dimensional coordinate system called Screen Space, ie. a bitmapped display. The Chart Geometry submodule supports calculations in a continuous (float) two-dimensional coordinate system, called Chart Space, a natural world space for simple scientific diagrams. In addition, the Geometry module provides affine mappings between the Chart and Screen spaces.

\subsubsection{Slate}

Slate [77] is a low level "device-indapendent" graphics package that has been ported to a number of Common Lisp platforms, window systems, and other graphics devices. It is intended to be used by developers of higher level scientific and statistical graphics systems rather than end users. Slate is something like a simplified version of CLX, ported to run on window systems and graphics devices other than X11.

Some of the design goals of Slate are: 
- It should be robust against errors in the calls from a higher level graphics system.

- It should be easy to port to a new window system, hardcopy device, or Lisp compiler.

- It should permit high perfomance implementations.

- Unless there's a good reason to do otherwise, the design should be as close to $X$ as possible, because we expect the $X$ port to be the most important.

The basic abstraction in Slate is) the slate. Slates are surfaces that can be drawn on and can receive input of various kinds. To make porting easy, the imaging model is fairly primitive; Slates are essentially bitmaps of some finite depth.

At present the set of drawing operations allows us to outline, fill, or tile simple geometric shapes like points, line segments, or polygons, draw characters and strings in a variety of fonts and colors, and copy rectangular sets of pixels from one slate to another. The behavior of all the drawing operations in Slate can be described in the following way: The drawing operation changes the values of a set of destination pixels in the slate. For each destination pixel, a source pixel value is calculated and the destination pixel is set to some simple function (at present some boolean combination of the bits) of the source and destination pixel values.

A typical drawing operation takes three types of arguments: a pen, which is an abstraction that encapsulates boolean operation, color, font, line style, etc., a slate to draw on, and a specification for the set of destination pixels. Sets of destination pixels are specified using the abstractions for discrete screen geometry provided by the Geometry package (see [75]).

Slate provides seperate abstractions for the more complex pen parameters, like colors, boolean operations, fonts, line styles, and tiling patterns.

There are two basic kinds of slates, standard, visible slates and invisible slates. All drawing operations work "in the same way" on both visible and invisible slates An invisible slate serves roughly the same purpose as a pixmap in $X$. It gives us a surface to draw on that can be copied rapidly to multiple places on one or more visible slates.

Another major abstraction in Slate in the screen. The screen object captures information about the device a slate is actually displayed on that isn't 
specific to any slate, such as, the number of bits per pixel. What color the user sees for a given pixel value is determined by the current colormap of the slate's screen.

\subsubsection{Chart}

Chart [76] is a simple example of a (slightly) higher level graphics package built on top of Slate. It is a quick and dirty approximation to $S$ style graphics $[4,5,6]$. What we mean by "S style graphics" is output-only, line and point plots, with labels and tic marks, where locations are specified in an arbitrary $2 \mathrm{~d}$ world coordinate system and the scaling to screen coordinates is done more or less automatically.

\subsubsection{Graph}

The Graph module [62] provides a simple protocol for data structures used to represent graphs (networks), graph nodes, and graph edges, and a sample implementation of that protocol.

\subsubsection{Browser}

The Browser module [62] provides a generic graph browser, implemented using the Clay and Graph modules and the graph layout methodology described below.

\subsubsection{Lisp-NPSOL Interface}

The NPSOL package [60] provides a number of functions for calling the Fortran optimization package NPSOL $[36,37,79,80,81]$ from Common Lisp, using the foreign function interface provided with Franz Allegro Common Lisp [29].

\subsubsection{Clay}

Clay [65] is the beginnings of a system designed to support drawing and interacting with $2 \mathrm{~d}$ (and 3d) pictures for visualizing scientific data, both the observational data that's the usual domain of statisticians and the computed "data" arising in computer experiments and algorithm animation. It 
provides a toolkit of standard plot components and mechanisms for pasting components together to make it easy for the user to improvise new kinds of plots. All plot components obey a clearly defined protocol. This means that there is a consistent user interface and that users can reliably define new types of plot components that serve in the existing user interface.

\subsubsection{Basic Math}

The Basic Math module [64] provides data structures and procedures implementing relatively low-level numerical analysis functionality. It is a building block for the Cactus module. Included in Basic Math are: machine constants, special functions (eg. beta, gamma) extended vector operations (analogous to the BLAS[49] used in Linpack[24]), evaluation and interpolation (eg. generic continued fractions) 1d numerical integration, and basic random number generators.

\subsubsection{Probability}

The Probability module [67] supports inference and Monte Carlo simulation (including bootstrapping) in a unified framework through a protocol for Probability-Measure classes. Probability measure objects are responsible for generating samples from themselves, computing their quantiles, and computing the probabilities of appropriate sets, including tail probabilities. The defined probability measure classes includes the standard oneand higher-dimensional parametric densities and discrete distributions, and non-parametric measures, either resulting from density estimates or the empirical measure of a set. (It's worth noting that simple descriptive statistics like mean, median, etc., are generic functions in the probability measure protocol and are applied to data sets by viewing them as empirical distributions.)

\subsubsection{Cactus}

Cactus [59] is a module implementing geometric abstractions for scientific computing as CLOS objects, discussed below. 


\section{Geometric Abstractions for scientific comput- ing}

Cactus is a system for numerical linear algebra and optimization implemented in CLOS. Cactus is designed to closely model the abstractions used in a course on finite dimensional (vector) spaces [83]. It provides representations for a variety of spaces, points (elements of some space), and mappings between spaces. The linear algebra core of Cactus deals with vector spaces, vectors, and linear transformations; more general spaces, points, and mappings are used are used to support constrained optimization at a similar level of abstraction [52].

The implementation of Cactus is, in part, an experiment to see how closely a program can follow natural mathematical abstractions, without losing the level of performance in large numerical problems provided by traditional Fortran subroutine libraries. To evaluate the results of this experiment, I have compared, in [59], the design, implementation, and performance of Cactus on certain standard numerical linear algebra problems with Linpack [24], a classic high-quality Fortran package for solving systems of linear equations and related problems.

The result of the experiment was that the overhead in using a high level of abstraction is modesi -- the runtimes of Cactus versions of common matrix decompositions range from about the same as the corresponding Linapck routines to perhaps about $50 \%$ longer. At the same time, the object-oriented design of Cactus eases code reuse and customization in ways that are not possible in Fortran.

\section{Declarative graph layout using nonlinear pro- gramming}

Automatic layout of graphs is problem that has a sizable literature; for recent surveys see [95]. Roughly speaking, methods for layout can be divided into procedural and declarative (also known as constraint-based [46]).

By procedural layout, we mean simply that there is a procedure, which, given a graph, computes its layout. A typical layout procedure (such as that given by Rowe et al. [87]) traverses the graph, assigning each node to an unoccupied site in a regular grid of allowed positions. The order of traversal and the method of site assigfiment are chosen in the hope of 
producing a "readable" layout: a minimal number of crossed edges, short (but not too short) edges, almost straight paths between important pairs of non-adjacent nodes, node positions whose $\mathrm{y}$ ( or $\mathrm{x}$ ) coordinates are consistent with the partial ordering of a directed graph, and so on. There are often additional passes over the graph during which the nodes are re-arranged in an attempt to improve the layout.

Declarative layout could be thought of as a special case of procedural layout, where the procedure definition is based on three basic abstractions:

- a domain of possible layouts (for a given graph),

- a specification "language" that allows the user to express essential and desirable features of the layout, and

- a generic solver, that takes a graph and a specification, and returns an element of the domain that meets or well approximates the specification.

For example, the domain might be all possible assignments of nodes to positions in a discrete lattice in the plane. The specification language might allow the user to ask to minimize some loss function (eg. the number of edge crossings) subject to constraints on the node positions (eg. no two nodes can occupy the same position in the lattice).

The distinction between procedural and declarative layout is, of course, very similar to the distinction between procedural and declarative programming languages. In particular, our notion of declarative layout is intentionally close to constraint programming languages $[10,96,50,51]$.

The advantage of procedural layout is speed. A procedural method makes one or a lew passes over the graph; the declarative example given above is an expensive integer programming problem, whose solution would require the equivalent of many iterations over the graph.

The advantage of the declarative approach is flexibility. In general, the only way for a user to modify the result of a procedural layout is to reprogram the procedure; because of the ad hoc nature of most layout procedures, it is difficult to know how to change the procedure to get the desired effect. The three abstractions of the declarative approach - the domain, the specification, and the solver - gives a use: immediate access to a whole class of layouts. The key research issue is to design abstractions to provide a large and useful class of layouts, without giving up too much in performance compared ad hoc procedural methods. 
Our proposal for declarative layout is an extension of the spring model [25, 46]. The spring model is based on a physical analogy: Imagine a spring placed between each pair of nodes in the graph. Let the rest length of the spring be the desired distance between the nodes, eg., proportional to the length of the shortest path in the graph connecting the two nodes. Then simply minimize the energy. (This is a minor variation on multidimensional scaling $[9,98]$.)

The domain of the layout is the space of node positions, $\oplus^{n} \Re^{2}$, that is, the $\mathrm{n}$-fold direct sum of the plane. The specification language is the set of rest lengths (and spring conitants). The solver is whatever minimization routine is used.

Our extension to the spring model is to minimize the energy subject to affine inequality (and equality) constraints on the node positions, in other words, nonlinear programming [28, 38, 52, 53]. For our solver, we are using standard packages for constrained minimization, in particular MINOS and NPSOL $[36,37,79,80,81]$. The possibility of affine constraints gives us a much richer specification language, making it easy, for example, to preserve the directedness of a graph in the vertical or horizontal ordering of the nodes, or to adjust a layout for the size and shape of the window in which the graph is being displayed.

The use of modern optimization methods, as represented by MINOS and NPSOL, gives us still greater flexibility. We can experiment, with other energy functions than the spring model and eventually provide users with more general ways to specify the energy than the rest lengths and spring constants. For example, we are considering energy functions that depend on the relationships of pairs of edges as well on pairs of nodes --. with the goal of minimizing or at least controlling edge crossings.

The following figures show examples of the use of this layout methodolgy, as implemented in the Browser package [62]. The first two figures ( 1 and 2) show the browser used as a programming tool:

The next three figures demonstrate alternate layouts of a single graph, using custom order and distance functions. They show a pedigree of Przewalski's horses [35]. The figures show 3 alternative layouts: (figure 3) treats the pedigree as an undirected graph, (figure 4) uses inheritance to place parents above children, and (figure 5) uses distance function based on kinship, the probable genetic similarity between two individuals. 


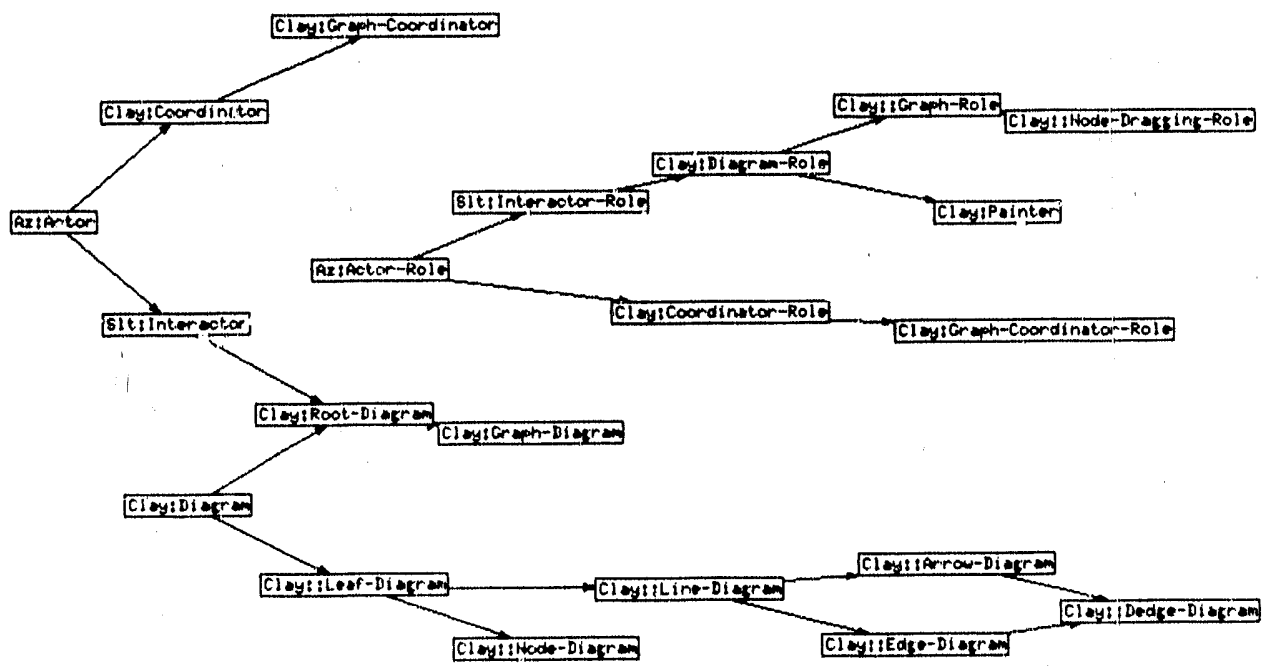

Figure 1: A class inheritance graph for some classes related to graph browsers.

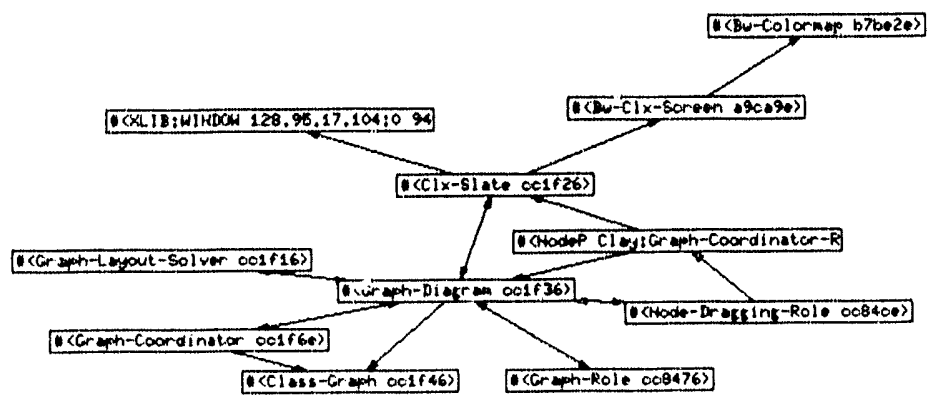

Figure 2: A examiner graph for some objecis used to make a class browser. 


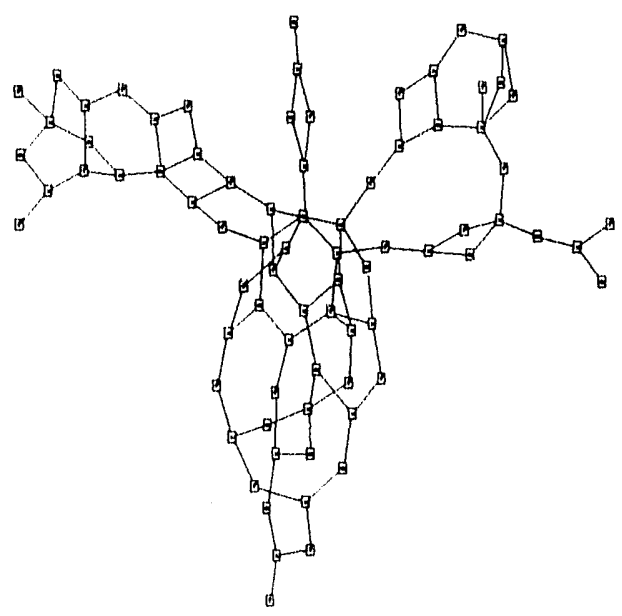

Figure 3: Horse pedigree layout, treating the podigree as an undirected graph.

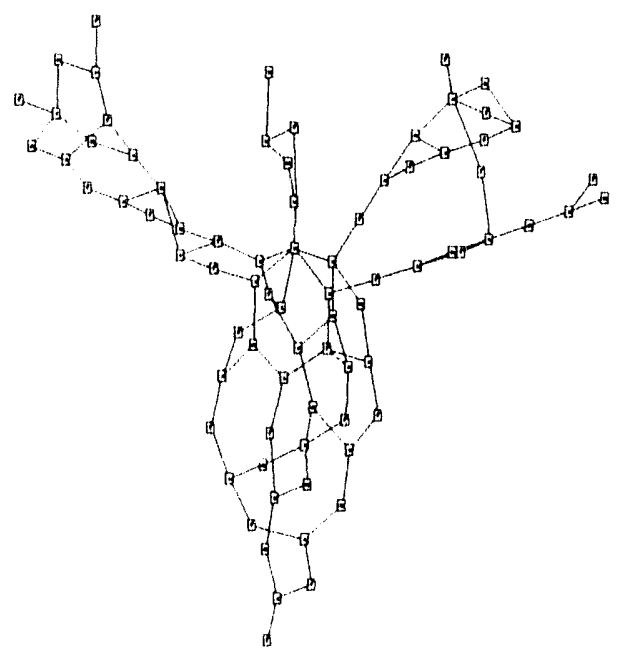

Figure 4: Horse pedigree layout using inheritance. 


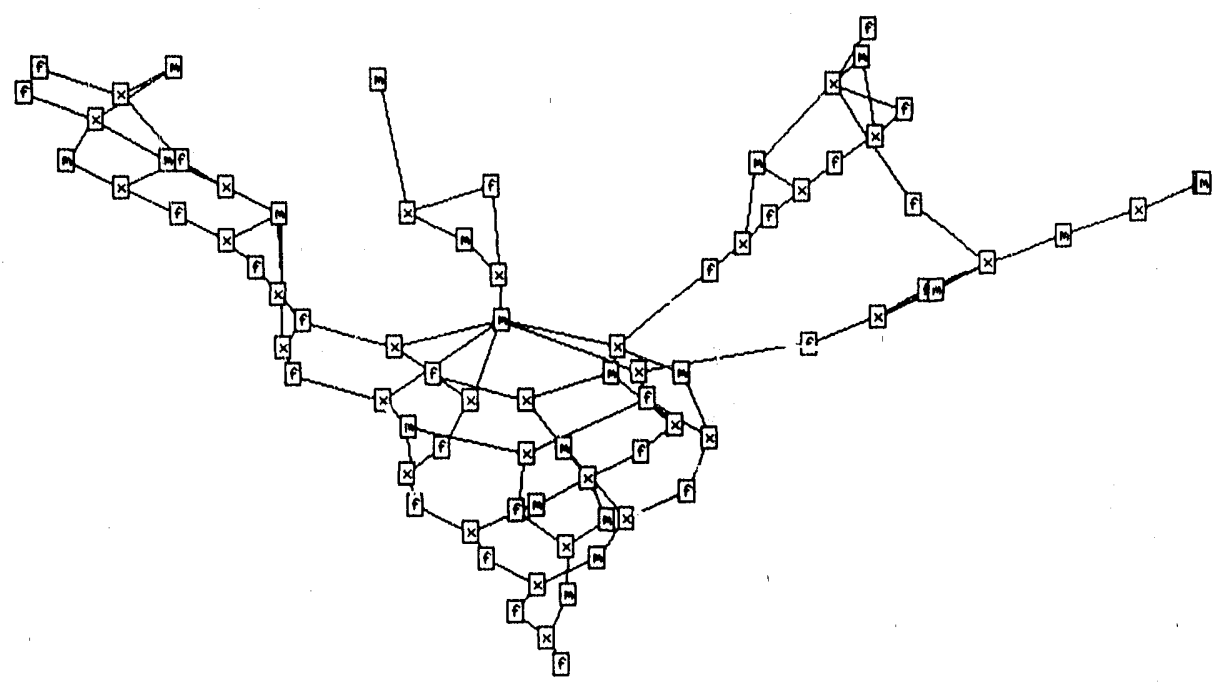

Figure 5: Horse pedigree layout using kinship and inheritance.

\section{Multiple Views of Data}

Most work in scientific visualization is concerned with high quality iendering of three-dimensional ohjects representing scalar or veitor valued functions of $R^{3}$. Displays are typically created in batch mode, and interaction with the displays is limited.

In contrast, research in statistical graphics has concentrated on methods for visualizing high-dimensional data, using real time motion and interaction. For an overview, see Cleveland and McGill [19]. We have recently abstracted out from this research two concepts, which we refer to as focusing and linking, that seem to unify much of this diverse methodology [15].

To display complicated information, like z large program, an automobile and all iis parts, or some multivariate statistical data, a common instinct is to draw a picture that is equally complicated, such as printing the program on the screen in a small font, rendering the automobile and its parts as transparent solids, or presenting the data as a tableau of Chernoff faces [17]. Attempts at such dense encoding are seldom successful. It is usually more effective to construct a number of simple, easy to understand displays, each focused clearly on a particular aspect of the underlying data.

Focusing techniques may involve selecting subsets, dimension reduction, or some more general manipulation of the layout of information on the page 
or screen. Examples of subset selection techniques are panning and zooming [13], and slicing [32]. Examples of dimension reduction techniques are projection and false coloring of multi-channel images [39, 40].

Techniques for more general layout manipulation include univariate data transformations, logical zooming (demonstrated in the accompanying video) [14], and a variety of techniques from human-computer interface research for adapting to a user's "focus of attention" or "point of interest"[54], such as generalized fisheye views [31, 26], Rooms [42], and Cone Trees [86].

Methods for focusing can be automatic, or interactive, or some combination of the two. An example of automatic focusing is exploratory projection pursuit as originally proposed by Friedman and Tukey [30]. The Prim-9 [27] and Orion $[58,55,56]$ systems provided interactive versions of projection pursuit.

A consequence of focusing is that each view will only convey partial information about the data. We can compensate for this fact by displaying multiple views. Multiple views, however, should not be regarded in isolation. They need to be linked so that the information contained in individual views can be integrated into a coherent image of the data as a whole.

How views can be linked depends on whether they are displayed in sequence over time, or in parallel, simultaneously.

The principal mechanism for linking views over time is through smooth change in the position of objects on the screen. Examples for this kind of linking are rotating three-dimensional point clouds and a higher dimensional generalization, the Grand Tour [3,11]. More generally, any smooth animation can be considered as a set of linked multiple views, spread out over time.

There are a number of techniques for displaying multidimensional point data through simultaneous multiple views that are linked by drawing lines connecting the points in the different views corresponding to the same observation. Examples are Andrews' plots [2], parallel coordinate plots [44, 97], and $m$-and-n plots [23]. The window "wiring diagrams" in Rooms [42] are an example of linking corresponding objects by lines in a very different context.

Painting multiple views is an alternative linking technique that is at least as effective for multidimensional point sets and is more easily generalized to other types of data. Painting multiple views integrates scattered information by marking corresponding parts of multiple displays with color (or some other form of highlighting). It is a generalization of "scatterplot brushing", 
whicin dates to the late 70 's $[82,58,56,78,7]$.

In a recent paper [15] and a related video [16] we demonstrate a number of graphical methods that are examples of focusing and linking in applications, including linguistics, geographic information systems, time series analysis, and the analysis of multi-channel images arising in radiology and remote sensing.

\subsubsection{Architecture for high performance user interfaces}

The need to solve concrete scientific and engineering problems is a tremendous stimulant of creativity, especially in ways of displaying and interacting with specialized kinds of information. It follows that an ideal system for visualization should help users to improvise new kinds of displays quickly, including new modes of interaction with those displays. Failing that, a good system should permit experts to implement small extensions of existing functionality quickly. For this to be true, it must be possible to implement a change that seems small to the user by a small change to the program. This implies that the abstractions used in the program must correspond closely to the way the users think about the underlying problem.

It may seem that we are belaboring an obvious point, but it is rare in scientific computing to find systems whose programming abstractions are at all similar to the underlying problem they are intended to solve.

Consider, for example, what we will call the "standard" implementation model for brushing scatterplots. In the standard implementation, each scatterplot has an $N \times 2$ array holding the $\mathrm{X}$ and $\mathrm{Y}$ coordinates of the points. This array is created typically by extracting two-dimensional records from an underlying database and applying a viewing transformation to get window coordinates. In addition, there is a color array of length $N$ that is shared by all plots. When brushing, the program examines the XY array to find the indices of the points under the mouse, changes, if necessary, the values in the corresponding locations in the color array, and redraws points at the coordinates in the corresponding locations in all the XY array.

This implementation strategy is natural for a programmer with experience in conventional scientific computing; Fortran subroutine libraries often represent important information purely by location in arrays. Nearly all implementations of brushing scatturplots of which we are aware, including our own early work, share the essential feature (and failing) of this model, which is that the correspondence between plots is represented by matching array 
indices. Unfortunately, it precludes even the most trivial extensions. For example, suppose the user wishes to simultaneously analyze three data sets: all the parients in a clinical trial of some drug, the male patients in the trial, and the female patients. Typical commercial implementations of brushing scatterplots either restrict the user to a single data set, or brush separate data sets separately, ie., brushing in a plot of all patients has no effect on plots of male patients or plots of female patients.

If we think of brushing scatterplots in terms of direct manipulation, then it's obvious what wrong with the "standard" implementation: There's no reliable representation for the identity of the underlying entity that's being brushed.

We next present an implementation model for painting multiple views based on direction manipulation. This model had its origins in the Antelope [57, 71], Plot Windows [91, 90, 92], and Data Viewer [12,43] systems. It is the basis for the Clay package, discussed in section 2.0.11.

To successfully support direct manipulation, our programming model has several components that are implicit in a conceptual model of direct manipulation:

- Subjects in a Database

There is an object (a Subject) in the programming environment representing each abstract entity (eg., a multispectral image pixel) that the user might want to examine, select with the mouse, and manipulate.

Each Subject has associated with it a set of Attributes; an Attribute has an identity independent of any particular Subject. We can evaluate an Attribute for any Subject for which it is defined and we can change the value for some, if not all, Attributes. We can also define now Attributes for a given Subject at runtime. This is similar to the data model proposed in [84].

Mutable Attributes are required to support the mutable appearances of the Subject's Presentations. Having an extensible set of Attributes allows different Presentations to behave differently in ways decided at runtime.

In some object-oriented programming languages, it is straightforward to represent Subjects by objects and Attributes by the slots (instance variables) of the Subject objects. Unfortunately, in many languages and databases, it may too expensive or not even possible to have mutable slots or to add slots at runtime. However, we can use essentially 
the same programming model as long as Subjects have unique identities and we can build mutable tables that map Subject identities to colors or other appearance parameters. We can then generalize our notion of Attribute to include both slots that are intrinsically part of each Subject and these mutable tables, defined at runtime.

The key points are that we can: (1) get values for any defined SubjectAttribute pair (2) set values for all Subjects and some Attributes and (3) define new mutable Subject-Attribute pairs at runtime.

Our programming model also presumes that Subjects can be organized into structured Collections, like the Collection classes of Smalltalk [41], eg. lists, directed graphs, or dictionaries. We also presume that new types of Collections can be defined - - if not by a random user, then at least by a programmer sophisticated enough to develop new kinds of plots.

- Presentations in a Scene

The Scenc consists of a directed acyclic graph (usually a tree) of ob jects (Scene Nodes) representing geometric shapes (including text), in a two or three dimensional Scene Space. The Sccne is rendered on to a display surface by standard computer graphics techniques. Some nodes in the Scene are visible representations or Presentations of some of the Subjects. (Our use of the word "Presentation" is similar, but not identical to [18].)

A Subject determines the position and appearance of its Presentations through an output Lens (see below). The hierarchical or graph structure of the Scene will often reflect a similar hierarchy in the Subjects; in other words, a Presentation of a Collection may have children that are Presentations of the elements of the Collection.

- Links

There must be a mechanism for linking Presentations and their Subjects. There are three kinds of connections between Presentations and Subjects:

- Lenses for output filtering

A Lens maps from Subjects to be visualized into draw-able colored geometric shapes (including text) in the Scene space; it computes the position and appearance of a Presentation from the Attributes of its Subject. 
In general, each Presentation could have its own unique Lens, computing position, shape, size, color, texture, etc., from its Subject however it likes. However, the standard painting operation--. and most of the interesting variations-can be implemented with a small number of simple Lenses, shared across Presentations and across plots as well.

- Input-Translators

An Input-Translator maps input events received by a Presentation to operations on its Subject.

- Consistency-Constraints

A Consistency-Constraint ensures that the Scenes always reflect the current state of the Subjects.

To implement a simple version of painting for scatterplots, we first suppose the Subjects (the records in the multivariate dataset) have $p$ quantitative Attributes and one color Attribute. We also suppose the records are organized into Data-Set collections; a Data-Set is essentially a list of records, with perhaps additional (summary) information about its records and Attributes.

In a scatterplot, the Scene is a tree. A Scatterplot-Root node typically has four children: a Label node that is a Presentation of a Data-Set, X-Axis and Y-Axis nodes, and a Point-Cloud node (another Presentation of the Data-Set). An Axis node has a Label node (a Presentation of a continuous Attribute) and a Ruler node as children; a Ruler has Tic and possibly TirLabel children. A Point-Cloud has Point-Glyph children, each of which is a Presentaticil of an element of the Data-Set.

A scatterplot uses a simple scatterplot Lens with two component sub-Lenses: (1) a Projection-Lens that maps a high dimensional record to a position in 2D Scene space and (2) a Color-Lens that reads the value in the color slot in the record object. The Projection-Lens varies from plot to plot, but is the same for all Point-Gilyph Presentations in a given plot. All the Point-Glyphs in all plots share the same Color-Lens, so that all Point-Glyphs always have the same color as their Subjects.

Painting input events received by a Point-Glyph are translated to the operation of changing the color Attribute of the glyph's Subject record. The Consistency-Constraint's ensure that all visible Point-Glyph's are redrawn when their Subject's color Attribute changes. 


\subsubsection{Image Painting}

As a test of our programming model, we have implemented a very demanding variation on the notion of painting multiple views: multichannel image painting. "Painting multiple views" means, in this context, that the user can interactively paint a representation of a pixel in any window, and all other representations of that pixel automatically take on the same color. For example, if the user sees a cluster in a scatterplot of channel space, it can be painted red to see where it falls in the image. Interesting objects in the image (eg. the brain or a known tumor in a medical image, a region with known land use classification in a satellite image, etc.) can be painted to see if they correspond to well defined clusters in channels space.

The key issue is squeezing out maximum performance, which is necessary for interactive response in even the smallest images. Our image painting implementation, based on the programming model described above, provides adequate interactive response with images up to size $1024 \times 1024 \times 6$ (Landsat TM) pixels.

\section{Reconstruction of two-dimensional manifolds from partial information}

The problem of constructing mathematical descriptions of objects given incomplete data is nearly ubiquitous in scientific and engineering applications. A simple example of this occurs when trying to fit a curve to a collection of data points. Although there exist good solutions for certain types of these reconstruction problems, an important class of reconstruction problems has received little attention. This class is characterized by the lack of organization of the input data. A protoypical problem in the class can be described as follows: given a relatively dense unordered set of points assumed to be distributed on or near some unknown "target" surface (such as a human heart or an automobile body) in three dimensions, construct a spline surface representation of the target. This problem arises naturally in many applications including medical imaging, machine vision, industrial engineering, and geometric modeling. Our proposed research intends to develop algorithms for a variety of reconstruction problems such as this. The principal difficulty is that the neither the topology nor the geometry of the surface are known a priori; both must be automatically inferred from the data points. 
We have invented an algorithm that has proven successful in estimating the topology of target surfaces ranging in complexity from a sphere to a complicated mechanical part of genus 3, from samples of sizes between 1000 and 10,000 . The algorithm also provides a crude approximation to the geometry of the surface. We also have experimented with methods of improving the geometry of the fit, using least squares techniques. Initial results have been encouraging. We have come up with a number of ideas for improvements of the fitting algorithms, which we still have to test. We also have ideas for mesh simplification, which should result in smoother and more parsimonious surface estimates. These have not yet been implemented. 


\section{$7 \quad$ Lectures}

\section{Refereed Talks at Conferences}

J.A. McDonald and W. Stuetzle. Data visualization using focusing and linking. Workshop on Size and Complexity in Information Worlds at CHI'91, New Orleans, April 28-29, 1991.

A.Buja, D. Haynor, J.A. McDonald, J. Michalak, Finbarr O'Sullivan, and W. Stuetzle. Graphical tools for quantitative radiology. IEEE 1990 Nuclear Science Symposium: Medical Imaging Conference, October 1990.

T. DeRose, T. Duchamp, H. Hoppe, J.A. McDonald, and W. Stuetzle. Determining Surface Topology from Unorganized Data. Second SIAM Conference on Geometric Design, Phoenix, Nov 4-8, 1991.

T. DeRose, T. Duchamp, H. Hoppe, J.A. McDonald, and W. Stuetzle. Fitting a Surface of Known Topology to Unorganized Data. Second SIAM Conference on Geometric Design, Phoenix, Nov 4-8, 1991.

\section{Invited Talks at Conferences}

J.A. McDonald and W. Stuetzle. Analysis of fish abundance in the Bering Sea: a case study in the use of graphical methods. American Statistical Association Annual Meeting, New Orleans, August 22 - 25, 1988.

W. Stuetzle. Statistical graphics. CBMS Research Conference on ComputerIntensive Methods, Washington DC, June 12-16, 1989.

W. Stuetzle. Examining associations between views of a data set. IMA conference on Robustness, Diagnostics, Computing, and Graphics in Statistics, Minneapolis, July 10 - September 1, 1989.

W. Stuetzle. A Comparison Between Lisp and S. IMA conference on Robustness, Diagnostics, Computing, and Graphics in Statistics, Minneapolis, July 10 - September $1,1989$.

J.A. McDonald and J. Pedersen. Geometric abstractions for constrained optimization of layouts. IMA conference on Robustness, Diagnostics, Computing, and Graphics in Statistics, Minneapolis, July 10 - September 1, 1989.

J.A. McDonald. Geometric abstractions in Scientific Computing. IBM Scientific Research Lab, Palo Alto, CA., October 1989.

J.A. McDonald and J. Pedersen. A case study of statistical computing in a Lisp environment: geometric abstractions for optimization, graph layoul, 
browsing and retrieval in large databases. American Statistical Association Annual Meeting, 1989.

J.A. McDonald. Geometric abstractions in scientific computing. Computer Science and Statistics, the 22th Symposium on the Interface, East Lansing, Michigan, May 1990.

A. Buja, J.A. McDonald, and W. Stuetzle: Painting multiple views: an interactive technique for "feeling" the shape of complex, multi-dimensional data. Invited Poster, Conference on Human Factors in Computing Systems, Seattle, April 1-5, 1990.

S. Weghorst and W. Stuetzie. Jeepers: an interface perception research tool. Invited Poster, Conference on Human Factors in Computing Systems, Seattle, April 1-5, 1990.

J.A. McDonald, J. Michalak, and W. Stuetzle. Interactive gruphics for treebased models. Biometrics Society ENAR Spring Meeting, Baltimore, April $1-4,1990$.

W. Stuetzle. Analysis of fish abundance in the Bering Sea - a case study in the use of graphical methods. IMS/WNAR. Western Regional Meetings, Bozeman, June 17-20, 1990.

J.A. McDonald, J. Michalak, and W. Stuetzle. Interactive graphics for treebased models. American Statistical Association Annual Meeting, Anaheim, August 5 - 9, 1990.

J.A. McDonald, J. Michalak, and W. Stuetzle. Focusing and linking as paradigms for the visualization of high-dimensional data and other complex objects. SoftStat '91 Conference on the Scientific Use of Statistical Software, Heidelberg, April 7-12, 1991.

W. Stuetzle. Principal Curves. Conference on Total Least Squares, Louvain, August 12-16, 1991.

J.A. McDonald, J. Michalak, and W. Stuetzle. Focusing and linking as paradigms for the visualization of high-dimensional data and other complex: objects. Seminar on Scientific Visualization, Schlos Dagstuhl, August 26--30, 1991. 


\section{Publications}

\section{Refereed Publications}

J.A. McDonald and J.O. Pedersen. Computing environments for data analysis III: Programming environments. SIAM Journal on Scientific and Statistical Computing, Vol. 9(2), pp. 380-400, 1988.

T. Hastie and W. Stuetzle. Principal curves. Journal of the American Statistical Association, Vol. 84, pp. 502-516, 1989.

J.A. McDonald. Object-oriented programming for linear algebra. SIGPLAN Notices Vol. 24 (10), pp. 175-184, 1989.

J.A. McDonald, W. Stuetzle, and A. Buja. Painting Multiple Views of Complex Objects. To appear in: Proceedings of the ECOOP/OOPSLA 1990 Conference.

A. Buja, J.A. McDonald, J. Michalak, and W. Stuetzle. Interactive data visualization using focusing and linking. In: Proceedings of Visualization'91, Oct 21-25, 1991, San Diego, CA.

A. Buja, J.A. McDonald, J. Michalak, W. Stuetzle, and S. Willis, Visualization of complex data. 17 minute video tape. In video proceedings of Visualization'91, Oct 21-25, 1991, San Diego, CA.

\section{Unrefereed Invited Publications}

J.A. McDonald. Interactive graphics for data analysis. In W.S. Cleveland and M.E. McGill, editors, Dynamic graphics for statistics, pp. 247-275. Wadsworth and Brooks/Cole, Belmont, CA, 1988.

J.A. McDonald. An outline of Arizona: a portable Lisp-based system for data analysis. In Proceedings of: Computer Science and Statistics, 20th Symposium on the Interface, Reston, Va., April 1988. (Also Technical Report 131, Dept. of Statistics, University of Washington)

W. Stuetzle. Inviter discussion of the article OMEGA - Online multivariate exploratory graphical analysis: routine search for structure by C. Weihs and H. Schmidli. Statistical Science, pp. 217-218, 1990.

W. Stuetzle. Odds Plots: A graphical aid for finding associations between views of a data sel. In Andreas Buja and Paul Tukey, editors, Computing and graphics in statistics, volume IMA 36. Springer-Verlag, NY NY, 1991.

J.A. McDonald and J.O. Pedersen. Geometric abstractions for constrained optimization of layouts. In Andreas Buja and Paul Tukey, editors, Compul- 
ing and Graphics in Statistics, volume IMA 36. Springer-Verlag, NY NY, 1991.

J.A. McDonald and J.O. Pedersen. A case study in statistical computing in a lisp environment: Geometric abstractiuns for constrained optimization of layouts. In Proceedings of the 1989 Joint Statistical Meetings, Statistical Computing Section, 1989.

\section{Technical Reports}

J.A. McDonald, W. Stuetzle, and A. Buja. Viewing high dimensional data by painting multiple views. Technical Report No. 174, Department of Statistics, University of Washington, 1989.

J.A. McDonald, W. Stuetzle, and A. Buja. Painting multiple vieus: an interactive technique for "feeling" the shape of complex, multi-dimensional data. Technical Report No. 178, Deptartment of Statistics, University of Washington, 1989.

M. Sannella. Analyzing Interactive Graphics Systems. Technical Report No. 182, Department of Statistics, University of Washington, 1989.

S. Weghorst and W. Stuetzle. Jeepers: An Interface Perception Research Tool. Technical Report No. 185, Department of Statistics, University of Washington, 1989.

J.A. McDonald and M. Sannella. Arizona overview and notes for release 0.0. Technical Report No. 202, Department of Statistics, University of Washington, 1991.

J.A. McDonald and M. Sannella. Geometry: a package for basic geometric calculation in Common Lisp, release 0.0. Technical Report No. 203, Department of Statistics, University of Washington, 1991.

J.A. McDonald and M. Sannella. Slate: a portable bitmap graphics package for Common Lisp, release 0.0. Technical Report No. 204, Department of Statistics, University of Washington, 1991.

J.A. McDonald and M. Sannella. Chart: a simple Common Lisp package for plotting data and functions, release 0.0. Technical Report no. 205, Department of Statistics, University of Washington, 1991.

T. DeRose, T. Duchamp, H. Hoppe, J.A. McDonald, and W. Stuetzle. Reconstructing two-dimensional manifolds from scattered data: Motivation and background. Technical Report No. 215, Depaprtment of Statistics, University of Washington, 1991. 
J.A. McDonald and M. Sannella. Geometry: a package for basic geometric calculation in Common Lisp, release 0.0. Technical ReportNo. 218, Department of Statistics, University of Washington, 1991.

J.A. McDonald. A simple graph browser. Technical Report No. 219, Department of Statistics, U/niversity of Washington, 1991.

J.A. McDonald. Definitions: a simple database for typesetting documentation. Technical Report No, 220, Department of Statistics, University of W'ushington, 1991.

J.A. McDonald and M. Niehaus. Announcements: an implementation of implicit invocation. Technical Report No. 221, Department of Statistics, University of Washington, 1991.

J.A. McDonald. Calling NPSOL from Common Lisp. Technical Report No. 222, Department of Statistics, University of Washington, 1991.

J.A. MCDonald and M. Sannella. Arizona overview and notes for release 1.0. Technical Report No. 223, Department of Statistics, University of Washington, 1991.

\section{Video Tapes}

W. Stuetzle. Odds Plots: examining associations between views of a data set. Video tape, 20 minutes; Department of Statistics, University of Washington, 1988.

W. Stuctzle. Analysis of fish abundance in the Bering Sea: a case study in the use of graphical methods. Video tape, 15 minutes; Department of Statistics, University of Washington, 1989.

A. Buja, J.A. McDonald, J. Michalak, W. Stuetzle, and S. Willis. Visualization of quantitative data. Video lape, 27 minutes; Department of Statistics, University of Washington, 1990.

\section{Submitted}

H. Hoppe, T. Derose, T. Duchamp, J.A. McDonald, and W. S'tuctzle. Surface reconstruction from unorganized points. Submitted to SiGiRAPH 9?.

\section{In Preparation}

J.A. McDonald. Actors, interactors, and coordinators. Technical Report. Department of Statistics, Iniversity of Washington, 1991. 
J.A. McDonald. Basic Math: a package of assorted numerical analysis functions in Common Lisp. Technical Report, Department of Statistics, University of Washington, 1991 .

J.A. McDonald. Clav: a high performance user interface toolkit. Technical Report, Department of Statistics, University of Washington, 1991.

J.A. McDonald. Image painting. Technical Report, Department of Statistics, University of Washington, 1991.

J.A. McDonald. Probability Measure Objects in CLOS. Technical Report, Department of Statistics, University of Washington, 1991.

J.A. McDonald and M. Sannelia. Arizona overview and notes for release 1.0. Technical Report, Department of Statistics, University of Washington, 1991.

J.A. McDonald and M. Sannella. Chart: a simple Common Lisp package for plotting data and functions, release 1.0. Technical Report, Department of Statistics, University of Washington, 1991.

J.A. McDonald and M. Sannella. Slate: a portable bitmap graphics packagr for Common Lisp, release 1.0. Technical Report, Department of Statistics. University of Washington, 1991.

J.A. McDonald and M. Sannella. Geometry: a package for basic geometrir: calculation in Common Lisp, release 1.0. Technical Report, Department of Statistics, University of Washington, 1991. 


\section{Research by Finbarr O'Sullivan}

O'Sullivan's work has focused on three areas. (i) Multi-dimensional Statistical Function Estimation, (ii) System Identification, and (iii) Image Analysis. Reseach products include: 8 articles in peer reviewed journals ( 5 published and 3 accepted), 5 manuscripts in journal review, 5 non-peer reviewed publications ( 4 proceedings and a discussion), 5 reviewed abstracts and several invited talks. In addition, O'Sullivan directed the $\mathrm{PhD}$ thesis work of Kevin Anderson. Kevin's work was partially supported by a $50 \%$ Research Assistantship from the grant.

\subsection{Multi-dimensional Statistical Function Estimation}

The work here was concerned with the development and analysis of approaches to non-parametric function estimation under a range of standard statistical models.

\section{Manuscripts}

1. O'Sullivan F., T'wo-dimensional Laplacian smoothing by iterative methods. J. Amer. Statist. Assoc., 85:213-219, 1990.

2. Cox D. D. and O'Sullivan F., Asymptotic analysis of penalized likelihood and related estimators. Ann. Statis., pages 1676-1695, 1990.

3. O'Sullivan F., Discrete Laplacian smoothing by Fonrier methods. J. Amer. Statist. Assoc, 86:634-642, 1991.

4. O'Sullivan F, Non-parametric Estimation in the Cox Proportional Hazards Model. Ann. Statis. (to appear), 1992.

5. O'Sullivan F, and Pawitan Y., Tomographic Density Estimation. J. R. Statist. Soc (B) (to appear), 1992.

6. O'Sullivan F., Comment on the MARS paper by Professor Friedman. Ann. Statis., pages $1676-1695,1990$.

7. Cox D. D. and O'Sullivan F., Generalized non-parametric regression. Technical Report, Department of Statistics, Iniversity of Washington 1990 . 


\section{Abstracts and Invited Talks}

1. O'Sullivan F., Multidimensional Smoothing Methods: Direct and Indirect. Proc. ASA Anual Meetings, Washington D.C., 1989.

2. O'Sullivan F., Multivariate Methods in the Analysis of Dynamic Emission Tomography Data. Workshop on Curve Estimation, Heilelberg 1991.

\subsection{System Identification}

Here the interest was in the estimation of distributed parameters in system identification problems. Initially the work concentrated on problems arising in reservoir modeling; more recently there is an increasing emphasis on examples arising in medical imaging. Anderson's thesis considers a non-linear deconvolution problem in arising in the analysis of episodic hormone data whose structure is closely related to the deconvolution problem arising in reflection seismology (Mendel Optimal Seismic Deconvolution, 1983).

\section{Manuscripts}

1. O'Sullivan F., Constrained non-linear least squares regularization with application to the estimation of functional parameters in elliptic partial differential equations. SIAM J. Numer. Anal., 1635-1649, 1990.

2. O'Sullivan F., Sensitivity Analysis for regularized estimation in som system identification problems. SIAM J. Sci. Stat. Comp., 1991.

3. Anderson $K$ and O'Sullivan F., An Efficient Statistical Deconvolution Method for Episodic: Hormone Data. Biometrics (submitted), 1991.

4. O'Sullivan F., Imaging radiotracer model parameters in PE'T: A mixture analysis approach. IEEE Trans. Med. Imaging (submitted), 1991.

\section{Abstracts and Invited Talks}

1. O'Sullivan F., Sensitivity analysis for system identification. STAM Special Meeting on Optimization, Boston, MA, 1989.

2. O'Sullivan F., Metabolic Image from Dynamic Emission T'omography Data. ASA 1991 Joint Anual Meetings, Atlanta, CA. 
3. O'Sullivan F., Pawitan Y. and Graham M.M., Metabolic Imaging Techniques for Dynamic PET Data. IEEE 1991 Nuclear Science Symposium: Medical Imaging Conference.

\subsection{Image Analysis}

During most of the funding period, the work in this area has been motivated by applications in emission tomography.

\section{Manuscripts}

1. O'Sullivan F., Wash C., Stewart M., and Motel C., Rain estimation from infrared and visual GOES statellite imagery. J. App. Meteor. 29:209-223, 1990 .

2. O'Sullivan F., Penalized likelihood-type estimation in pet and more general inverse problems involving linear operators. Ann. Statist. (under revision) 1992.

3. Pawitan Y and O'Sullivan F., Data dependent bandwidth selection for' emission computed tomography reconstruction. IEEE Trans. Med. Imaging (submitted), 1991.

4. O'Sullivan F. Pawitan Y. Haynor D., A comparison of least squares and maximum likelihood image reconstruction in emission computed tomography. IEEE Trans. Med. Imaging.(submitted), 1991.

5. O'Sullivan F., Mixture estimation with multichannel image data. J. Amer. Stat. Assor. (to appear), 1992.

\section{Abstracts and Invited Talks}

1. O'Sullivan F., Smooth mixture estimation with multichannel image data. Proc. 2.3'rd Symp. Interface of Computer Science and Statistics, 1990 .

2. McDonald J., Michalak J., O'Sullivan F., Stuetzle W., Haynor D., and Buja A., Graphical tools for quantitative radiology. IEEE 1990 Nuclear Science Symposium: Medical Imaging Conference. 
3. O'Sullivan F, Pawitan Y, Harrison RL, Lewellen TK., PET reconstruction: Improving Least Square Solutions. IEEE 1990 Nuclear Science Symposium: Medical Imaging Conference.

4. O'Sullivan F., Inverting the Attenuated Radon Transform. Workshop on Empirical Processes and Statistics, Oberwolfach 1991. 


\section{Research by Kevin Anderson}

Kevin Anderson completed a Ph.D. thesis: "Efficient Deconvolution of Episodic Hormone Data". A summary of this work is given below. A manuscript (cited in O'Sullivan's report) has been submitted related to this work.

\section{Summary of Thesis}

Endocrine time series data often reveal episodes of punctuated hormone secretion. Joint estimation of the number, temporal location, and amplitude of these pulses, together with secretion and clearance rates, is an important practical problem for researchers in this field. This dissertation introduces a new stochastic model for hormone concentration in peripheral blood and presents two methods to estimate its parameters. Pulses result from a marked sequence of secretory events which are cleared by a physiologically reasonable kinetic model. Various characterizations of the noise are possible. Several renewal processes are used to model secretory event occurrences. A new estimation procedure, based on pseudo maximum likelihood, is applied to simulated responses stemming from these processes. These studies show that joint estimation of the mean rate and amplitude of events, together with the error variance and rate of clearance is possible but that strong correlations exist between estimates. A second procedure, called EZ, is implemented to estimate the number and location of individual events together with pulse characteristics and the level of noise in the response. A generalized cross-validation statistic governs the model selection process. The algorithm is shown to perform remarkably well on simulated and actual data. It is efficient enough to permit calculation of bootstrap variance estimates for all quantities, including the number of pulses in the series. 


\section{Research by Michael Sannella}

Michael Sannella is working on applications of constraint programming to user ointerface design.

During the past year, he has continued studying constraint programming, graphics and user interfaces, and worked at applying these techniques to the development of interactive data analysis systems. By observing the limitations of existing data analysis systems, he has discovered several areas where constraint technology could be used to make these systems more flexible and useful. He developed a plan for creating a constraint-based datia analysis system, and described it in a PhD thesis proposal which was approved in early 1991. Since then, he has worked on implementing pieces of such a system.

His thesis proposal identified several tasks performed by a data analysis system that could be better addressed using constraint technology. These include the tasks of laying out multiple plots on a display screen, specifying the user interface to interactive plots, and managing the numerous data sets and plots created during an interactive data analysis session. He has developed a number of programs to investigate possible solutions to these problems. One program allowed a data analyst to control the connections between data sets and plots by visually editing a data flow graph. Another program addressed the issue of specifying and customizing layout programs by using constraints to compare multiple layouts. This work has lead him to investigate the more general problem of debugging constraint networks, research which would be applicable to any constraint system.

He has attended several conferences on areas related to my work, which gave him an opportunity to talk with other people working in the fields of user interfaces, graphics, and statistical data analysis environments. In Spring 1990, he went to the ACM Special Interest Group on ComputerHuman Interaction (CHI'90), the main conference on user interface issues. In Fall 1990, he attended the Seminar on User Interface Systems and Technology (U1ST'90), a conference concentrating on the problems of programming complex user interfaces. In Spring 1991, he attended the Symposium on the Interface between Computer Science and Statistics (Interfaco'91), as well as the Western Computer Graphics Symposium.

Finally, he has done basic software support work in a number of areas. He installed new versions of Allegro Common Lisp on our Sun system, investigating and reporting bugs and installing patches. He ported several 
programs from $\mathrm{C}$ to Lisp, including an implementation of the Simplex algorithm and a general three-dimensional affine geometry package. Ho wrote several programs to translate MRI image files to a format allowing them to be analyzed. He has also been the local contact person for the "Garnet" user interface development system, retrieving and installing several versions of the software, giving talks and demos, and helping other people on campus interested in the system. 


\section{Research by Steve McKinney}

Steve McKinney is currently working on his dissertation under the preception of Werner Stuetzle. His topic is "automatic painting of high dimensional point clouds of data so as to reveal structure through low dimensional views". Suppose we collect data on many (more than three) variables in the course of a study or experiment. Since we cannot visualize data in more than 3 dimensions, we are always forced to look at our data variables in pairs using scatterplots or in triples using point cloud rotation or stereo vicwing. But, in so doing, we lose information because we cannot tell which data point in one scatterplot corresponds to which data point in the other scatterplots for a given member of the data set. In order to understand an individual element of the data set (or a group of individuals) in the context of the full dimensional space from which the individual element arose, we need to be able to connect each data point corresponding to that individual member (or connect all the points corresponding to a group) across all the lower dimensional views. This prompted the development of scatterplot brushing and painting computer techniques whereby the analyst can color a data point or set of data points in one scatterplot, and the computer then colors the corresponding data points in all the other scatterplots. However, the analyst is still restricted to viewing low dimensional scenes of the high dimensional structure, and painting features as seen in these low dimensional views. Often structures of interest in high dimensiunal spaces do not generate obvious or interesting patterns when projected onto lower dimensional surfaces. 'The goal of Steve's dissertation is to extend this principle and create an algorithm which will detect "non-gaussian" (i.e. interesting) structure in the full dimensional data point cloud, then color the structure and choose appropriate low dimensional views so as to highlight the structure and allow the analyst to understand it in the context of the full dimensional space from whence the data came.

Initially Steve experimented with structure detection using the ACE algorithm. If variables are correlated (i.e. have some kind of functional relationship), the $\mathrm{ACE}$ algorithm will find transformations of the variables so as to maximize the linear correlations among the transformed variables. He investigated the potential of these $\mathrm{ACE}$ transformations for detecting structure and suggesting coloring schemes to highlight the structure. The transformations obtained via the ACE algorithm were not specific enough to suggest coloring schemes which effectively rovealed the data structures to 
the analyst.

Subsequently he has been studying Human Visual Perception theory and experiments to suggest alternate strategies. These readings have helped shape his current paradigm. Initially he searches for clustering in the data. Spatially isolated portions of the data need to be examined individually. Subsequently he attempts to understand the topological features of each separate cluster in the data. The most effective coloring strategies depend on the topology of the structure which is to be visualized. He is thus experimenting with clustering algorithms, as well as algorithms for discerning topological features of data point clouds. He has recently combined the Data. Viewer graphical package (developed by Andreas Buja) with clustering packages (developed by John Michalak) to form the graphical viewport for the Automatic Painting package. Over the next year he will continue to research issues in topological feature identification, perceptually effective coloring schemes, and perceptually effective graphical display techniques for high dimensional data. 


\section{Bibliography}

\section{References}

[1] Gul Agha. Actors: A model of concurrent computation in distributed. systems. MIT Press, Cambridge, MA, 1986.

[2] David F. Andrews. Plots of high-dimensional data. Biometrics, 28:125 $136,1972$.

[3] D. Asimov. The grand tour: a tool for viewing multidimensional data. SIAM Journal of S'cientific and Statistical Computing, 6:128-143, 1985.

[4] R.A. Becker and J.M. Chambers. Design of the $S$ system for data analysis. $C A C M, 27(5): 486-495,1984$.

[5] R.A. Becker and J.M. Chambers. S: An Interactive Environment for Data Analysis and Graphics. Wadsworth, Belmont, Ca., 1984.

[6] R.A. Becker, J.M. Chambers, and A.R. Wilks. The New S Language. Wadsworth and Brooks/Cole, Pacific Grove, CA, 1988.

[7] R.A. Becker and W.S. Cleveland. Brushing scatterplots. Technometrics, 29:127-142, 1987.

[8] Daniel Bobrow, David Fogelsong, and Mark Miller. Definition Groups: Making sources into first-class objects. In Bruce Shriver and Peter Wegner, editors, Rescarch Directions in Object-Oriented Programming. MI'T Press, Cambridge MA, 1987.

[9] I. Borg and J. Lingoes. Multidimensional Similarity Structure Analysis. Springer-Verlag, New York, 1987.

[10] Alan H. Borning, Robert A. Duisberg, Bjorn Freeman-Benson, Axel Kramer, and Michael Woolf. Constraint Hierarchies. In Proceedings of the 1987 ACM Conference on Object-Oriented Programming Systems, Languages, and Applications, pages 48-60. ACM, October 1987.

[11] Andreas Buja and Daniel Asimov. Grand Tour methods: an outline. In Computer Science and S'tatistics: Proce. 17th Symp. on the Interface, pages 63-67, Amsterdam, 1986. Elsevier. 
[12] Andreas Buja, Catherine Hurley, and John Alan McDonald. A Data Viewer for multivariate data. In Computer Science and Statistics: Proc. 18th Symp. on the Interface, pages 171-174, Washington, D.C., 1987. ASA.

[13] Andreas Buja, Catherine Hurley, and John Alan McDonald. Elements of a viewing pipeline for data analysis. In W.S. Cleveland and M.E. McGill, editors, Dynamic Graphics for Statistics. Wadsworth and Brooks/Cole, Belmont, Ca., 1988.

[14] Andreas Buja, John A. McDonald, John Michalak, Werner Stuetzle, and Steve Willis. Visualization of quantitative data, 1990. A 27 minute video tape; Dept of Statistics, U. of Washington.

[15] Andreas Buja, John Alan McDonald, John Michalak, and Werner Stuetzle. Interactive data visualization using focusing and linking. In Gregory M. Nielson and Larry Rosenblum, editors, Proceedings of the Second IEEE Conference on Visualization, San Diego, 22-25 October 1991. IEEE Computer Society Press, 1991.

[16] Andreas Buja, John Alan McDonald, John Michalak, Werner Stuetzle, and Steve Willis. Visualization of complex data. In Video Procecdings of Visualization'91, 1991. 17 minute video tape.

[17] Herman Chernoff. The use of faces to represent points in k-dimensional space graphically. Joumal of the American Statistical Assoriation, 68:361-368, 1973.

[18] E.C. Ciccarelli. Presentation based user interfaces. Technical Report 794, MIT AI Laboratory, 1984.

[19] W.S. Cleveland and M.E. McGill. Dynamic Graphics for Statistics. Wadsworth and Brooks/Cole, Belmont, Ca., 1988.

[20] Tony D. DeRose. Geometric programming: a coordinate-free approach. ACM SIGGRAPH Tutorial Course No. 25, 1988.

[21] Tony D. DeRose. A coordinate-free approach to geometric programming. In W. Strasser, editor, Theory and Practice of Geometric Modeling. Springer Verlag, 1989. 
[22] Tony D. DeRose. A coordinate-free approach to geometric programming (a geometric algebra and its implementation). Technical report, Dept. of Computer Science, U. of Washington, 1989. course notes for CSCI557, Spring 1989.

[23] Persi Diaconis and Jerome H. Friedman. $\mathrm{M}$ and $\mathrm{N}$ plots. In Recent Advances in Statistics: Papers in Honor of Herman Chernoff on his Sixtieth Birthday, pages 425-447. Academic Press, New York, 1983.

[24] J.J. Dongarra, C.B. Moler, J.R. Bunch, and G.W. Stewart. LINPACK Users' Guide. SIAM, Philiadelphia, 1979.

[25] P. Eades. A heuristics for graph drawing. Congressus Numerantium, 42:149-160, May 1984.

[26] Kim M. Fairchild, Stephen E. Poltrock, and George W. Furnas. SemNet: three-dimensional graphic representations of large knowledge bases. In Raymonde Guindon, editor, Cognitive Science and its applications for human-computer interaction, chapter 5, pages 201-233. Lawrence Erlbaum Associates, Hillsdale, NJ, 1988.

[27] Mary Anne Fisherkeller, Jerome H. Friedman, and John W. Tukey. Prim-9, an interactive multidimensional data display and analysis system. In Proc. of Pacific 75, ACM Regional Conference., 1974.

[28] R. Fletcher. Practical Methods of Optimization. Wiley, Chichester, Second edition, 1989.

[29] Franz, Inc. Allegro CL, An Extended Common Lisp, User's Guide, Release 4.0. Franz Inc., Berkeley, CA, January 1991.

[30] Jerome H. Friedman and John W. Tukey. A projection pursuit algorithm for exploratory data analysis. IEEE Trans. Comput., C-23, 1974.

[31] George W. Furnas. Generalized fisheye views. In Proceedings of CHI'S6, Human Factors in Computing Systems, New York, 1986. ACM.

[32] George W. Furnas. Dimensionality constraints on projection and section views of high dimensional loci. In Computer Science and Statistics: Proc. of the 20 Symp. on the Interface, Washington, D.C., 1988. ASA. 
[33] David Garlan and Ehsan Ilias. Low-cost, adaptable integration policies for integrated environments. In Proceedings of ACM SIGSOFT'90:Fourth Symposium on Software Development Environments, pages 1-10, December 1990.

[34] David Garlan and David Notkin. Formalizing design spaces: Implicit invocation mechanisms. In Proceedings of VDM'91: Formal Software Development Methods, 1991.

[35] Charles Geyer. personal correspondence, 1989.

[36] Chilip E. Gill, Sven J. Hammarling, Walter Murray, Michael A. Saunders, and Margaret H. Wright. User's guide for LSSOL (version 1.0): a Fortran package for constrained linear least squares and convex quadratic programming. Technical Rejort SOL 86-1, Systems Optimization Laboratory, Dept. of Operations Research, Stanford, 1986.

[37] Philip E. Gill, Walter Murray, Michael A. Saunders, and Margaret II. Wright. User's guide for NPSOL (version 4.0): a Fortran package for nonlinear programming. Technical Report SOL 86-2. Systems Optimization Laboratory, Dept. of Operations Research, Stanford, 1986.

[38] Philip E. Gill, Walter Murray, and Margaret H. Wright. Practical Optimization. Academic Press, London, 1981.

[39] Alan R. Gillespie, Anne B. Kahle, and Richard E. Wa'ter. Color Enhancement of Highly Correlated Images. I. Decorrelation and HSI Con+ trast Stretches. Remote Sensing of Environment, 20:209-235, 1986.

[40] Alan R. Gillespie, Anne B. Kahle, and Richard E. Walker. Color Enhancement of Highly Correlated Images. II. Channel Ratio and "Chromaticity" Transformation Techniques. Remote Sensing of Environment, 22:343-365, 1987 .

[41] A. Goldberg and D. Robson. Smalltalk-80, The Language and Its Implementation. Addison-Wesley, Reading, MA, 1983.

[42] D. Austin Henderson, Jr, and Stuart K. Card. Rooms: The use of multiple virtual workspaces to reduce space contention in a window-based graphical user interface. ACM Transactions on Graphics, 5(3):211-243, July 1986. 
[43] Catherine Hurley. The Data Viewer: a program for graphical data analysis. PhD thesis, Dept. of Statistics, U. of Washington, 1987.

[44] A. Inselberg. The plane with parallel coordinates. The Visual Computer, 1:69-91, 1985.

[45] Ira Kalet, Christine Sweeney, and Jonathan Jacky. Software design for interactive graphic radiation treatment simulation systems. In Proceedings of the Fourteenth Annual Symposium on Computer Applications in Medical Care, pages 594-598, Washington, D.C., November 1990. IEEE Computer Society Press.

[46] Tomihisa Kamada. On visualization of abstract objects and relations. $\mathrm{PhD}$ thesis, University of Tokyo, 1988.

[47] Mark Kantrowitz. Portable Utilities for Common Lisp, User Guide and Implementation Notes. Technical Report CMU-CS-91-143, School of Computer Science, CMU, 1991.

[48] Leslie Lamport. Latex, a Document Preparation System. AddisonWesley, Reading, MA, 198.5.

[49] C. Lawson, R. Hanson, D. Kincaid, and F. Krogh. Basic linear algebra subprograms for fortran usage. ACM TOMS, 5(3):308-371, 1979.

[50] Wm Leler. Constraint Programming Languages. Addison-Wesley, Reading, MA, 1988.

[51] D. Levitt. Machine tongues X: Constraint languages. Computer Music Journal, 8:9-21, 1984 .

[52] David G. Luenberger. Optimization by vector space methods. Wiley, NY NY, 1969.

[53] David (i. Luenberger. Linear and Nonlinear Programming. AddisonWesley, Reading, MA, second edition, 1984.

[54] Jock D. Mackinlay, Stualt K. Card, and George G. Robertson. Kapid controlled movement through a virtual 3d workspace. Computer Graphics, $24(4): 171-176,1990$.

[55] John Alan McDonald. Exploring data with the Orion I workstation, 1982. A 25 minute, $16 \mathrm{~mm}$ sound film, which demonstrates programs 
described in McDonald (1982) It is available for loan from: Jerome H. Friedman, Computation Research Group, Bin \# 88, SLAC, P.O. Box 4349, Stanford, California 94305.

[56] John Alan McDonald. Projection pursuit regression with the Orion I workstation, 1982. a 20 minute, $16 \mathrm{~mm}$ color sound film, which demonstrates programs described in McDonali (1982) It is available for loan from: Jerome H. Friedman, Computation Research Group, Bin \# 88, SLAC, P.O. Box 4349, Stanford, California 94305.

[57] John Alan McDonald. Antelope: data analysis with object-oriented programming and constraints. In Proc of the 1986 Joinl Statistical Meetings, Stat. Comp. Sect., 1986.

[58] John Alan McDonald. Interactive graphics for data analysis. In W.S. Cleveland and M.E. McGill, editors, Dynamic Graphics for Statistics, pages 247-275. Wadsworth and Brooks/Cole, Belmont, CA, 1988. Ph.1). thesis, Dept. of Statistics, Stanford, June 1982.

[59] John Alan McDonald. Object-oriented programming for linear algebra. SIGPLAN Notices (Proceedings OOPSLA'89), 24(10):175-184, 1989.

[60] John Alan McDonald. Calling NPSOL from Common Lisp. Technical report, Dept. of Statistics, U. of Washington, October 1991.

[61] John Alan Mclonald. Definitions: a simple database for typesetting documentation. Technical report, Dept. of Statistics, U. of Washington, October 1991.

[62] John Alan McDonald. A simple graph browser. Technical report, Dept. of Statistics, U. of Washington, October 1991.

[63] John Alan McDonald. Actors, interactors, and coordinators. 'Technical report, Dept. of Statistics, U. of Washington, 1992. in preparation.

[64] John Alan McIDonald. Basic Math: a package of assorted numerical analysis functions in Common Lisp. Technical report, Dept. of Statistics, U. of Washington, 1992. in preparation.

[65] John Alan McDonald. Clay: a high performance user interface toolkit. Technical report, Dept. of Statistics, U. of Washington, 1992. in preparation. 
[66] John Alan McDonald. Image painting. Technical report, Dept. of Statistics, U. of Washington, 1992. in preparation.

[67] John Alan McDonald. Probability Measure Objects in CLOS. Technical report, Dept. of Statistics, U. of Washington, 1992. in preparation.

[68] John Alan McDonald and Mark Niehaus. Announcements: an implementation of implicit invocation. Technical report, Dept. of Statistics, U. of Washington, October 1991.

[69] John Alan McDonald and Jan O. Pedersen. Computing environments for data analysis I: Introduction. SIAM J. Scientific and Statistical Computing, 6(4):1004-1012, 1985.

[70] John Alan McDonald and Jan O. Pedersen. Computing environments for data analysis II: Hardware. SIAM J. Scientific and Statistical Computing, 6(4):1013-1021, 1985.

[71] John Alan McDonald and Jan O. Pedersen. Computing environments for data analysis III: Programming environments. SIAM J. Scientific and Statistical Computing, 9(2):380-400, 1988.

[72] John Alan McDonald and Jan O. Pedersen. A case study in statistical computing in a lisp environment: Geometric abstractions for constrained optimization of layouts. In Proc. of the 1989 Joint Statistical Meetings, Stat. Comp. Sect., 1989.

[73] John Alan McDonald and Jan O. Pedersen. Geometric abstractions for constrained optimization of layouts. In Andreas Buja and Panl Tukey, editors, Computing and Graphics in Statistics, volume IMA 36. Springer-Verlag, NY NY, 1991.

[74] John Alan McDonald and Michael Sannella. Arizona overview and notes for release 1.0. Technical report, Dept. of Statistics, U. of Washington, October 1991.

[75] John Alan McDonald and Michael Sannella. Geometry: a package for basic geometric calculation in Common Lisp, release 1.0. Technical report, Dept. of Statistics, U. of Washington, October 1991.

[76] John Alan McDon.del and Michael Sannella. Chart: a simple Common Lisp package for plotting data and functions, release 1.0. Technical report, Dept. of Statistics, U. of Washington, 1992. in preparation. 
[77] John Alan McDonald and Michael Sannella. Slate: a portable bitmap graphics package for Common Lisp, release 1.0. Technical report, Dept. of Statistics, U. of Washington, 1992. in preparation.

[78] John Alan McDonald, Werner Stuetzle, and Andreas Buja. Painting multiple views of complex objects. SIGPLAN Notices, 25(10):245--257, 1990. (Proceedings OOPSLA/ECOOP'90).

[79] Bruce A. Murtagh and Michael A. Saunders. Large-scale linearly constrained optimization. Mathematical Programming, 14:41-72, 1978.

[80] Bruce A. Murtagh and Michael A. Saunders. A projected Lagrangian algorithm and its implementation for sparse nonlinear constraints. Mathematical Programming Study, 16:84-117, 1978.

[81] Bruce A. Murtagh and Michael A. Saunders. MINOS 5.1 users' guide. Technical Report SOL 83-20R, Systems Optimization Laboratory, Dept. of Operations Research, Stanford, 1987.

[82] C. M. Newton. Graphics: from alpha to omega in data analysis. In P.C.C. Wang, editor, Graphical Representation of Multivariate Data. Academic Press, New York, 1978. Proceedings of the Symp. on Graphical Representation of Multivariate Data, Naval Postgraduate School, Morterey, Ca., Feb 24, 1978.

[83] Walter Noll. Finite-Dimensional Spaces (Algebra, Geometry, and Analysis), volume l. Martinus Nijhhoff, Dordrecht, 1987.

[84] R.W. Oldford. Object-oriented software representations for statistical data. Journal of Econometrics, pages 227-246, 1988.

[85] Steven P. Reiss. Connecting tools using message passing in the Field environment. IEEE Software, 7(4):57-66, July 1990.

[86] George G. Robertson, Jock D. Mackinlay, and Stuart K. Card. Cone Trees: animated 3d visualizations of hierarchical information. In Proceedings of CHI'91, Human Factors in Computing Systems, New York, 1990. ACM.

[87] L. A. Rowe, M. Davis, E. Messinger, C. Meyer, C. Spirakis, and A. Tuan. A browser for directed graphs. Software Practice and Experience, 17(1):61-76, January 1987. 
[88] Mark G. Segal. Programming Language Support for Geometric Computations. PhD thesis, Computer Science, U.C. Berkeley, 1989.

[89] G.L. Steele. Common Lisp, The Language. Digital Press, second edition, 1990.

[90] Werner Stuetzle. Design and implementation of plot windows. In Proceedings of the Statistical Computing Section of the American Statistical Association, pages $32-40,1987$.

[91] Werner Stuetzle. Plot windows. JASA, 82:466-475, 1987.

[92] Werner Stuetzle. Plot windows, 1987. A 25 minute video tape; Dept of Statistics, U. of Washington.

[93] Kevin J. Sullivan and David Notkin. Reconciling environment integration and component independence. In SIGSOFT'90: Fourth Symposium on Software Development Environments, Irvine CA, pages 208 225, June 1990 .

[94] Kevin J. Sullivan and David Notkin. Behavioral relationships in objectoriented analysis. Technical Report 91-09-03, Dept. of Computer Science and Engineering, U. of Washington, 1991.

[95] Roberto Tamassia, Giuseppe Di Battista, and Carlo Batini. Automatic graph drawing and readability of diagrams. IEEE Transactions of Systems, Man, and Cybernetics, 18(1):61-78, 1988.

[96] Pascal Van Hentenryck. Constraint Satisfaction in Logic Programming. MIT Press, Cambridge, MA, 1989.

[97] Edward J. Wegman. Hyperdimensional data analysis using parallel coordinates. JASA, 85(411):664-675, 1990.

[98] Forest W. Young. Multidimensional Scaling: History, Theory, and Applications. Lawrence Erlbaum Assoc., Hillsdale, NJ, 1987. edited by Robert M. Hamer. 

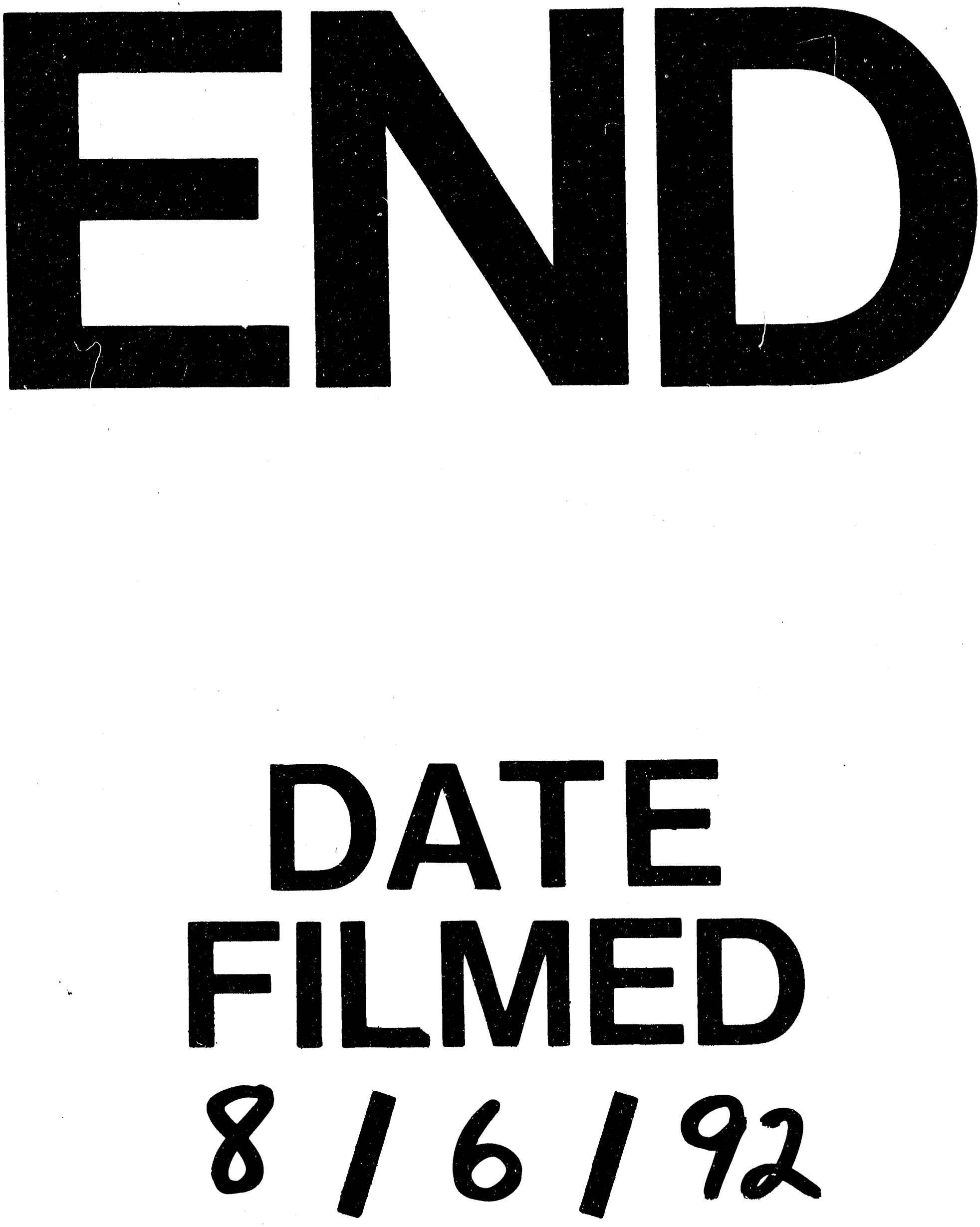

1 
